\title{
CLASSICAL PATHS AND SEMICLASSICAL GHOSTS
}

\author{
F. HAAKE \\ Fachbereich Physik, Universität GII Essen, 45117 Essen, Germany
}

\section{Dedicated to Prof. Iwo Biatynicki-Birula on the occasion of his 60th birthday}

The semiclassical approximation for the quantum propagator of the kicked top is shown to involve not only classical periodic orbits but also complex generalizations thereof. Such ghost trajectories have complex actions $S$ and thus contribute exponentially small terms as $\hbar \rightarrow 0$. However, close to bifurcations $\operatorname{Im} S$ can be very small whereupon ghosts become quite visible.

PACS numbers: $03.65 . \mathrm{Sq}, 05.45 .+\mathrm{b}$

This talk is based on work done in collaboration with D. Delande, B. Eckhardt, and M. Kuś. We are happy to discuss our work at the meeting in honor of Iwo Biatynicki-Birula, to whom we express our heartiest congratulations and best wishes.

Before telling the ghost story promised in the title, I would like to address a few personal words to Iwo. I could name many reasons for my respect for Iwo. One is the independence of his thinking; when, for instance, Iwo lectures about nonlinear variants of Schrödinger's equation for massive particles or about a Schrödinger equation for a photon, the audience is not dragged along a beaten path but experiences the curiosity of a very original :esearcher. Quite a different achievement of Iwo's is his Institute of Theoretical Physics at the Polish Academy of Sciences here in Warsaw. That Institute has provided a scientific home to a group of highly talented physicists. Like Iwo himself, his younger colleagues at the Institute could easily have found much better paid positions in prestigious physics departments abroad. Instead, and however inconvenient or even hard life may have been here at times, they chose to stick together in a country where they felt they were needed and where they belong; in a city where they had grown up and wanted their children to grow up; in an intellectual community which had formed them and which they wanted to keep blossoming. The Institute, now called Centre of Theoretical Physics is beautiful testimony of a combination of practical patriotism and scientific excellence which indeed has my highest esteem. Finally, I do not hesitate to express my admiration of the life that Iwo and his wife Sophie are leading, in solid and loving companionship through good days as well as hardship. 
Periodic orbits have long since been considered the tangible structure of the phase space of nonintegrable systems, the structure around which chaotic trajectories wind their largely unpredictable course [1]. More recently, the set of classical periodic orbits has been seen to serve as a backbone to (the trace of) the quantum mechanical propagator, at least in the semiclassical limit of strong excitation [2]. Even a formally exact representation can be given to the quantum propagator by summing contributions from classical orbits, provided the notion of a trajectory is generalized so as to allow for explorations of a phase space doubled in dimension by complexification [3]; such excursions are associated, e.g., with tunneling phenomena, i.e. penetration of phase space regions inaccessible to real classical trajectories.

A particularly noticeable and noteworthy influence of complex solutions of real classical equations of motion on quantum behavior arises when a dynamical system is steered through a bifurcation. In a tangent bifurcation, for instance, a pair of (real) classical orbits appear when some control parameter $\kappa$ is raised above a critical value $k_{c}$; for $k<k_{c}$ that pair usually lurks outside of, but close to the classical world as a pair of complex "ghosts" $[4,5]$.

From a semiclassical point of view complex periodic orbits can make no more than exponentially small contributions to the (trace of the) propagator. This is because such ghosts have complex actions, $S=\operatorname{Re} S+\mathrm{i} \operatorname{Im} S$, such that their semiclassical contributions $\exp (\mathrm{i} S / \hbar)$ acquire the magnitude $\exp (-\operatorname{Im} S / \hbar)$ which indeed decays exponentially with $\hbar \rightarrow 0$ (the wrong $\operatorname{sign}$ of $\operatorname{Im} S$ never seems to become physically relevant). However, as $k \rightarrow k_{\mathrm{c}}$ from below one has $\operatorname{Im} S \rightarrow 0$ and the two limits $\hbar \rightarrow 0$ and $k \rightarrow k_{\mathrm{c}}$ can conspire to impart quite considerable weight to the ghosts.

Gutzwiller's theory accounts for real periodic orbits only. These appear in the stationary-phase approximation of Feynman's path integral representation of the trace of the propagator. When complex ghosts visit, as precursors of real periodic orbits at an imminent bifurcation, they make saddle-point contributions to the path integral which can greatly improve the semiclassical approximation.

Right at a bifurcation further improvements become necessary. As is well known [6], the naive saddle-point and stationary-phase approximations diverge at a bifurcation. That failure is due to the vanishing of quadratic fluctuations of the paths admitted from the path extremizing the action. To get a meaningful semiclassical approximation at or very close to $k=k_{\mathrm{c}}$ one must therefore include at least cubic fluctuations. A tangent bifurcation thus contributes a term involving an Airy function.

In order to present the above ideas in more concrete terms we now turn to periodically driven systems. Their stroboscopic period-to-period dynamics is described by a quantum map of the form

$$
\psi_{n}=F \psi_{n-1}=F^{n} \psi_{0}
$$

Here $\psi_{n}$ is the state vector after $n$ periods of the driving; it is unitarily related to $\psi_{n-1}$ by the so-called Floquet operator $F$. The standard semiclassical approxima- 
tion for the trace of the propagator now takes the form [5]:

$$
\operatorname{tr} F^{n} \underset{\hbar \rightarrow 0}{\longrightarrow} \sum_{\substack{\text { periodic orbit. } \\ \text { period } n}} A \mathrm{e}^{\mathrm{i} S / \hbar}
$$

All real periodic orbits of period $n$ contribute here with their actions $S$ and $A$ related to their stability. The very structure of the right-hand side in (2) suggests to look at the one-sided Fourier transform with respect to the "variable" $1 / \hbar$,

$$
T_{n}(\omega)=\int_{0}^{\infty} \mathrm{d}\left(\frac{1}{\hbar}\right) \mathrm{e}^{-\mathrm{i}\left(\omega-\mathrm{i} 0^{+}\right) / \hbar} \operatorname{tr} F^{n},
$$

which should display, in its real part, a sharp peak whenever the real frequency $\omega$ coincides with the action $S$ of a periodic orbit; the weight of such a peak should be proportional to the amplitude $A$.

We have undertaken calculations of $T_{n}(\omega)$ for a model system, a periodically kicked top $[4,5,7]$. The specific Floquet operator chosen can be expressed in terms of the components of an angular momentum vector $J$ as

$$
F=\mathrm{e}^{-\mathrm{i} \frac{k}{2 j} J_{x}^{2}} \mathrm{e}^{-\mathrm{i} \frac{\pi}{2} J_{x}} .
$$

Obviously, this $F$ accounts for a rotation about the $x$ axis by $\pi / 2$ followed by a nonlinear rotation about the $z$ axis by an angle proportional to $J_{z}$. The squared angular momentum is conserved and yields a quantum number $j$ as

$$
J^{2}=j(j+1), \quad \text { with } j=\frac{1}{2}, 1, \frac{3}{2}, \ldots
$$

A IIilbert space of dimension $(2 j+1)$ pertains to a fixed value of $j$ and thus yields representations of $F$ by $(2 j+1) \times(2 j+1)$ matrices. As $j$ grows large, semiclassical behavior is attained. In fact, we may take $j$ as a dimensionless measure of $1 / \hbar$. In the classical limit, $j \rightarrow \infty$, the top is described by the classical unit vector $\lim _{j \rightarrow \infty} J / j$ whose end points range on the unit sphere as a phase space; for $k \gg 1$ that phase space is dominated by chaotic trajectories [6].

After numerically diagonalizing $F$ for all integer values of $j$ in the interval $1 \leq j<200$ we have determined the traces $\operatorname{tr} F^{n}$ for $n=1,2, \ldots$ Upon adapting the Fourier transforms (3) to the case of discrete $j$ as

$$
T_{n}(\omega)=\frac{1}{M} \sum_{j=j_{0}}^{j_{0}+M-1} \mathrm{e}^{-\mathrm{i} \omega j / M} \operatorname{tr} F^{n}
$$

we have obtained spectra such as the ones depicted in Fig. 1 for $n=1$ and a sequence of three values of $k$. Of the four peaks in Fig. 1a $(k=12)$ three appear precisely at the actions of fixed points of the classical dynamics. The second peak from the left in Fig. 1a, however, corresponds to no (real) classical fixed point. Upon increasing $k$ to 14 we find the spectrum $T_{1}(\omega)$ depicted in Fig. 1c where the "quantum peak" has split into two and now both of these have a classical fixed point as a partner. Classically, one has crossed a tangent bifurcation at $k_{\mathrm{c}}=12.73$. Therefore, had we chosen $M$ large enough, already the case $k=13$ depicted in Fig. 1b should resolve a doublet of peaks to which the one in Fig. 1a is a quantum precursor. 

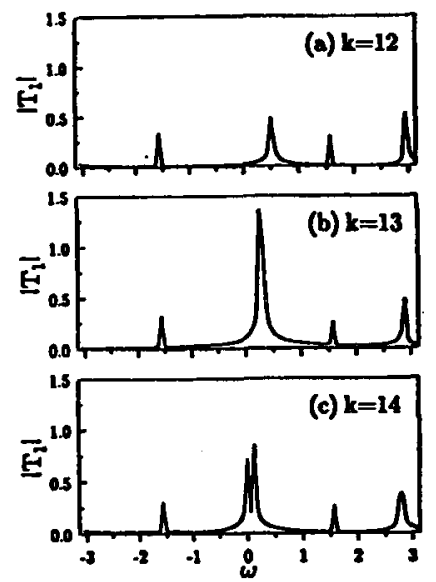

Fig. 1. Fourier transformed trace $T_{1}\left(\omega ; j_{0}=1, M=100\right)$ of the Floquet operator of the kicked top for various values of the control parameter $k$ : (a) below $k_{c}=12.73$ with a ghost peak at $\omega=0.51$ and three peaks corresponding to real periodic orbits; (b) slightly above $k_{c}$ where the highest peak corresponds to an unresolved doublet due to two real periodic orbits; and (c) sufficiently far above $k_{c}$ where the doublet is resolved.

The quantum peak in Fig. 1a may be interpreted as due to a complex solution of the real fixed-point equation. Such a "ghost orbit" can indeed be found for $k<k_{\mathrm{c}}$ and for $k=12$ turns out to have the complex action $S=0.51+0.02 \mathrm{i}$. A glance at Fig. 1a reveals $\operatorname{Re} S=0.51$ as the location of the peak in question. Moreover, we have checked the height of that peak to decay with increasing $j_{0}$ as $\exp \left(-0.02 j_{0}\right)$, i.e. with a rate equal to $\operatorname{Im} S$. In brief, the frequency dependence of $T_{1}(\omega)$ in Fig. 1 quantitatively conforms to a semiclassical description (2), provided the saddle-point contribution of the ghost is accounted for at $k=12$.

When looking at the $T_{n}(\omega)$ with larger values of $n$ and the corresponding classical equations for period- $n$ solutions we have found it not difficult to detect further ghosts, i.e. to spot classical bifurcations in the quantum propagator. It is not even necessary to hit, in choosing $k$, the immediate neighborhood of a bifurcation value since $\operatorname{Im} S$ appears to grow rather slowly as $k_{\mathrm{c}}-k$ increases.

A closer look at the integral representation of $\operatorname{tr} F^{n}$ will help to understand the ghosts' high visibility. For simpli.ity, we again choose $n=1$ and consider

$$
\operatorname{tr} F=\int \mathrm{d} x A(x) \mathrm{e}^{\mathrm{i} j S(x, k)},
$$

where $x$ is a real coordinate. For the kicked top such an expression arises $[4,5]$ when coherent-state teclniques are employed in evaluating $\operatorname{tr} F$; the coordinate $x$ may then be $x=\cos \phi \tan (\theta / 2)$ where the angles $\theta$ and $\phi$ determine the classical angular momentum vector as $J_{z} / j=\cos \theta,\left(J_{x}+\mathrm{i} J_{y}\right) / j=\exp (\mathrm{i} \phi) \sin \theta$; a second coordinate, $y=\sin \phi \tan (\theta / 2)$, has already been integrated over in order to arrive at (7). For $k$ near a tangent bifurcation one may expand the action $S$ in powers of 
$x-x_{\mathrm{c}}$ and $k-k_{\mathrm{c}}$. Putting $x_{\mathrm{c}}=0$ one has

$$
S=S_{0}+S_{x k} x\left(k-k_{\mathrm{c}}\right)+\frac{1}{2} S_{x x k} x^{2}\left(k-k_{\mathrm{c}}\right)+\frac{1}{6} S_{x x x} x^{3}+\ldots
$$

To determine fixed points one sets $\partial S / \partial x=0$. Note that the term $x^{2}\left(k-k_{c}\right)$ secures $x=0$ to be a doubly degenerate fixed point at $k=k_{\mathrm{c}}$. If $S_{x k}<0$ two real fixed points arise for $k>k_{c}$ while below the bifurcation there is a pair of complex conjugate ghosts. To leading order in $k-k_{\mathrm{c}}$ one gets $x_{\mathrm{s}}= \pm\left[2 S_{x k}\left(k_{\mathrm{c}}-k\right)\right]^{1 / 2}$. At that value of $x$ the integrand has a stationary phase if $k>k_{\mathrm{c}}$ and a saddle point if $k<k_{\mathrm{c}}$. The imaginary part of the ghost's action now comes out as

$$
\operatorname{Im} S \sim\left(k_{\mathrm{c}}-k\right)^{3 / 2} \text { for } k \leq k_{\mathrm{c}} .
$$

The exponent $3 / 2$ in the foregoing power law explains why ghosts are easy to detect: $\operatorname{Im} S$ increases rather slowly as $k_{\mathrm{c}}-k$ is raised from zero.

On both sides of the bifurcation the integral (7) draws a contribution from $x_{s}$ which is proportional to $\left.\left(\partial^{2} S / \partial x^{2}\right)^{-1 / 2}\right|_{x=x_{s}}$. It follows, as is well known and has already been mentioned above, that the naive stationary-phase or saddle-point approximation to (7) breaks down when $k \rightarrow k_{\mathrm{c}}$. Right at and very near $k_{\mathrm{c}}$ one may instead drop $S_{x x k} x^{2}\left(k-k_{c}\right)$ from (8) since it is smaller by a factor $\sim \sqrt{k-k_{c}}$ than the terms with $S_{x k}$ and $S_{x x x}$. One is then lead to a well known diffraction catastrophe integral which contributes to $\operatorname{tr} F$ in terms of the Airy function as [6]:

$$
2 \pi A\left(x_{s}\right)\left(2 / S_{x x x}\right)^{1 / 3} j^{1 / 6} \Lambda \mathrm{i}\left(S_{x k}\left(2 / S_{x x x}\right)^{1 / 3}\left(k-k_{c}\right) j^{2 / 3}\right)
$$

which interpolates between the saddle-point and the stationary-phase result as $k$ is raised from well below to well above $k_{\mathrm{c}}$.

Ghosts are not a peculiar property of just the kicked top. Recently, Van der Veldt, Vassen, and Hogervorst [8] have seen one in their spectra of He atoms in strong magnetic fields. Scharf and Sundaram have ascertained them for the periodically kicked rotator [9]. Main and Wunner [10] say their calculated spectra of the $\mathrm{H}$ atom in a magnetic field abound of ghosts related to various types of bifurcation. Stöckmann [11] is planning to locate bifurcations by varying the shape of his microwave resonators. And, of course, in the light of Balian's [3] old ideas all of quantum $\mathrm{n}$ echanics appears as a ghost story.

\section{References}

[1] H. Poincaré, Les Méthodes Nouvelles de la Mécanique Celeste, Tome I, Gautiers-Villars, Paris 1892.

[2] M.C. Gutzwiller, Chaos in Classical and Quantum Mechanics, Springer, New York 1990.

[3] R. Balian, G. Parisi, A. Voros, Phys. Rev. Lett. 41, 1141 (1978); R. Balian, in: Discourses in Mathematics and its Applications, No. 1, Eds. S.A. Fulling, F.J. Narcowich, (CDepartment of Mathematics, Texas A\&M University, College Station, Texas 1991.

[4] M. Kuś, F. Haake, D. Delande, Phys. Rev. Lett. 71, 2167 (1993).

[5] M. Kuś, F. Haake, B. Eckhardt, Z. Phys. B 92, 221 (1993). 
[6] M.V. Berry, C. Upstill, in: Progress in Optics, Ed. E. Wolf, North-Holland, Amsterdam 1980, p. 257; A.M. Ozorio de Almeida, J.H. Hannay, J. Phys. A 20, 5873 (1987); A.M. Ozorio de Almeida, Hamillonian Systems: Chaos and Quantization, Cambridge University Press, Cambridge 1988.

[7] F. Haake, Quantum Signalures of Chaos, Springer-Verlag, Berlin 1991.

[8] T. Van der Veldt, W. Vassen, W. Hogervorst, Europhys. Lett. 21, 903 (1993).

[9] R. Scharf, B. Sundaram, private communication.

[10] J. Main, G. Wunner, private communication.

[11] H.-J. Stöckmann, private communication. 